\title{
INTERACTING NUCLEI IN DISTANT GALAXIES
}

Wei Zheng, University of Alabama, U.S.A.

and

Steven A. Grandi, National Optical Astronomy Observatories, U. S. A.

The interaction of galaxies not only occurs in galactic scales, but also may be linked to the binary cores in active galactic galaxies. The presence of a binary in the center of galaxies was suggested by Begelman, Blandford and Rees (1980). Gaskell (1983) suggests that supermassive binaries may account for the observed structure of emission line profiles such as double peaks displaced by a significant velocity difference. Collin-Souffrin, et al. (1986) argue that line emission may be formed in the outer part of an accretion disk. The resultant profile, as expected from rotational motion, would be very broad and often possess a double-horn shape. However, the emission line profiles in most active galactic nuclei do not share such a resemblance, and there are only two reported cases, 3C390.3 (Pérez et al. 1987) and Arp102B (Chen, Halpern and Filippenko 1989), in which the broad Balmer line profile may be of such a shape. Therefore, the assumption for accretion disk is to be verified with care.

The N-galaxy 3C 390.3 has been monitored spectroscopically since 1974 (Osterbrock, Koski and Phillips 1975; Oke 1988). From various archives and literature, it is found that the Balmer lines change their intensities and profiles in a dramatic manner. The $\mathrm{H} \alpha$ profile is very broad (FWHM $\approx 15000 \mathrm{~km} \mathrm{~s}^{-1}$ ) and peculiar, and the relative intensities of its two humps (see figure 1) changes consistently with time, possibly periodically. Before 1980, the blue hump was significantly stronger than the one in the red. From 1980 to 1983 the blue hump became stronger (see Oke 1988). After 1983 the $\mathrm{H} \alpha$ profile has returned to its early shape and seems to have completed a full circle. Unlike the rapid (on the order of a month or even less) and aperiodic variation in the continuum and integrated line intensities, the change in broad profile seems slow and consistent.

Taking the analogy of cataclysmic variables: the double-horn profiles have been observed in cases of interacting stars. For example, the emission lines, both in He II and hydrogen Balmer lines in GD 552 (Stover 1985) show double-horn profiles and periodical changes in their line profiles, including the change in ratios of two humps. It is understood that the 
that the D-wave components (Smak 1976) are the signature of an emitting disk and the $\mathrm{S}$-wave component is from the emission at a hot spot which rotates and results in a moving component in the velocity space. the mass flow from the nearby interacting star provides the stream toward the core of a neutron star or white dwarf. Therefore, it is proposed that the variation of broad line profiles observed in $3 \mathrm{C} 390.3$ may be the result of a pair of interacting massive cores. The rotational velocity dominates and produces a variable double-horn profile. However, the line widths observed in broad line radio galaxies are one order larger than that in interacting stars. The Balmer decrements imply a much smaller density $\left(10^{10-12} \mathrm{~cm}^{-3}\right)$ than that in the cataclysmic variables. The much larger velocity and much thinner density make it unlikely that the broad line emission is simply formed in an accretion disk. We postulate that a significant rotational motion is involved.

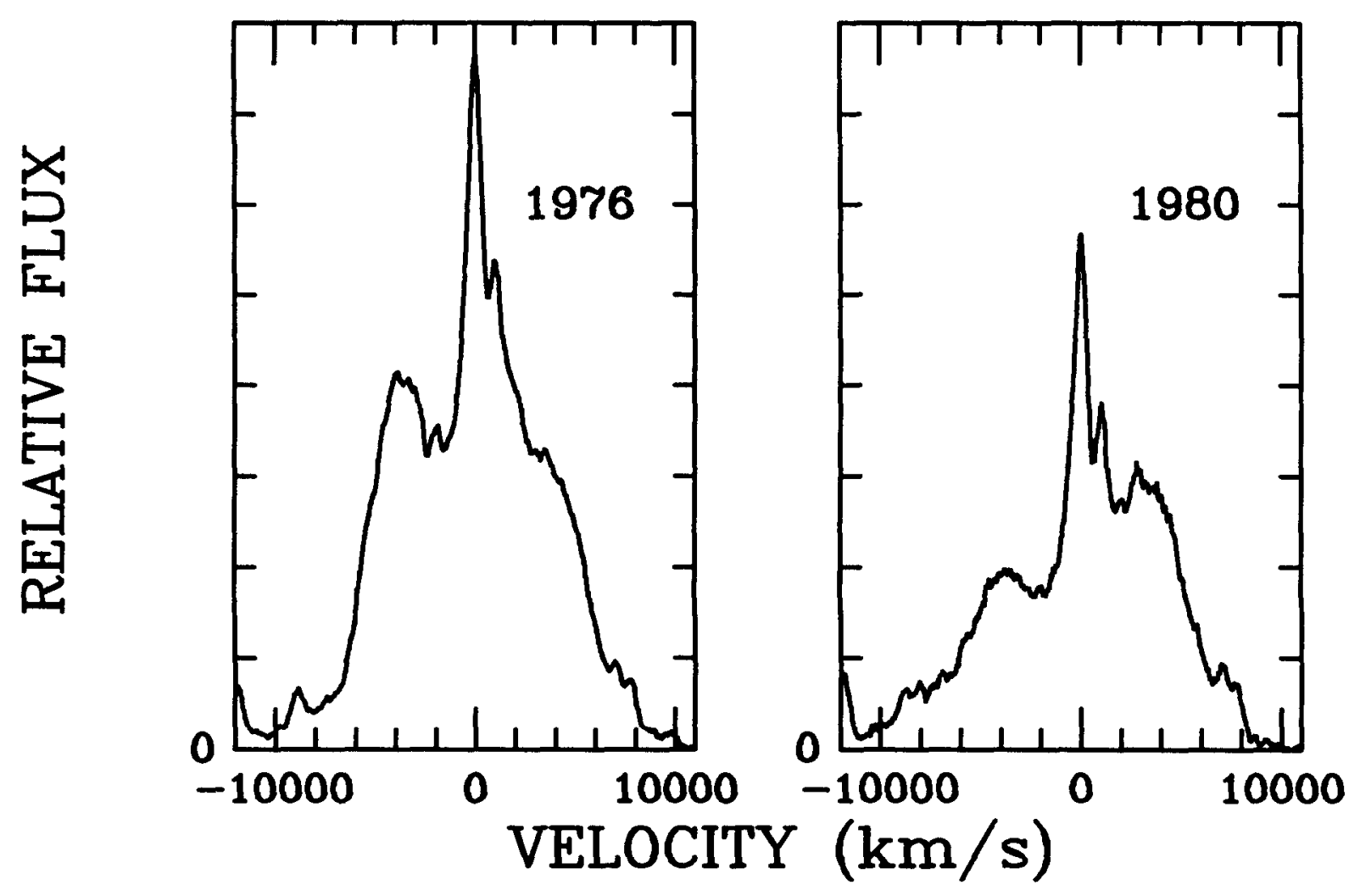

Figure 1, The $\mathrm{H} \alpha$ profile 
If the observed "squared" profiles are indeed due to the rotational velocity field, one can naturally explain their rare occurrence: only in the cases of double cores, the gas around the central engine has low enough velocity to be confined within the Roche lobe and engaged in a rotational motion which dominates. In other cases where only a single core is present, either the radiative or gravitational fields are radial and make it difficult to form a rotating line-emitting region. Thus, the profiles most commonly observed in extragalactic objects are logarithmic. Furthermore, it is possible to deduce the central mass from the rotational period and the distance to the central engine. Previous estimates can be made only by the order of magnitude. Preliminary result indicates that for $3 \mathrm{C} 390.3$ the value is $7 \times 10^{7} M_{\odot}$.

\section{REFERENCES}

Begelman, M. C., Blandford, R. D., and Rees, M. J. 1980, Nature, 287, 307.

Chen, K., Halpern, J. P., and Filippenko, A. V. 1989, Ap. J., 339, 742.

Collin-Souffrin, S., Dumont, S., Joly, M., and Péquignot, D. 1986, Astr. Ap., 166, 27.

Gaskell, C. M. 1983, in Quasars and Gravitational Lenses, Proc. 24th Liège Int. Ap. Coll. (Liège: Institut d'Astrophysique, Université de Liège), p. 473.

Oke, J. B. 1987, in Superluminal Radio Sources, eds. J. A. Zensus, and T. J. Pearson, (Cambridge: Cambridge University Press), p. 267.

Osterbrock, D. E., Koski, A. T., and Phillips, M. M. 1975, Ap. J. (Letters), 197, L41. . 1976, Ap. J., 206, 898.

Pérez, E., Penston, M. V., Tadhunter, C., Mediavilla, E., and Moles, M. 1988, M.N.R.A.S., 230, 353.

Smak, J. 1976, Acta Astr., 26, 277.

Stover, R. J. 1985, in Cataclysmic Variables and Low-Mass X-ray Binaries, ed. D. Q. Lamb and J. Patterson (Dordrecht: Reidel), p. 379. 